spontaneous chiral symmetry breaking, and is found throughout nature.

Now, Erio Tosatti and colleagues have predicted that two nested nanotubes sliding past one another should lead to chiral symmetry breaking. The researchers, who are based at SISSA, the ICTP and the Democritos National Simulation Center, all in Trieste, showed that even though both nanotubes are perfectly symmetric and achiral, they can be made to rotate with respect to each other. Moreover, this rotation reflects nanoscale physics that is not present in the oscillating string example. Specifically, the rotations suddenly increase or decrease as a function of sliding speed, and a phenomenon known as umklapp scattering also affects the rotation frequencies.

Although the sliding speeds used by Tosatti and colleagues were very high (of the order of hundreds of meters per second) the simulations may nevertheless prove relevant to a variety of potential nanomechanical systems, such as nanomotors.

\section{TOXICITY ASSAYS}

Reality check

Small doi: 10.1002/smll.200801788 (2009)

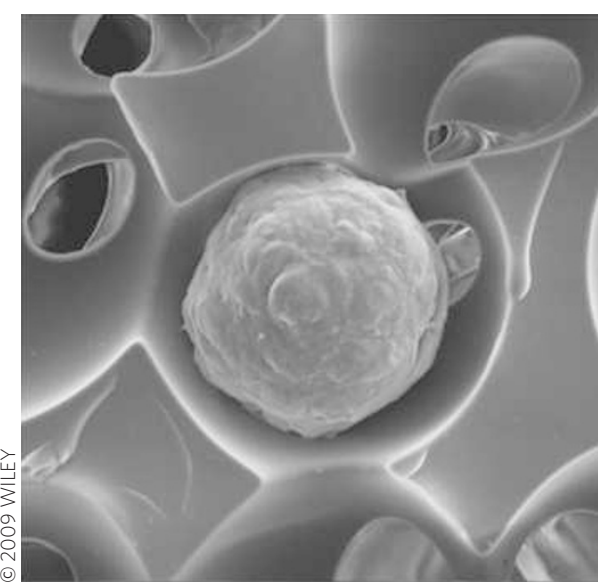

Two-dimensional (2D) cell cultures, which involve growing cells on a flat surface, are useful for quickly screening various biological effects including the toxicity of nanoparticles on cells. However, it is becoming increasingly clear that results from these cultures do not match those from animal studies. One reason is because the $2 \mathrm{D}$ culture models do not adequately recapitulate the three-dimensional (3D) organization of cells and tissues in the body. Researchers at the University of Michigan now show that a 3D cell culture model may improve the predictive power of current in vitro assays.

Nicholas Kotov and co-workers prepared a 3D liver tissue model by seeding liver cells on a porous scaffold made by using glass beads and polyacrylamide hydrogel. Cadmium telluride and gold nanoparticles were tested in this 3D tissue model, and compared with a conventional 2D cell culture - fewer changes in cell shape were seen in the 3D model. When the toxic effects of the nanoparticles were evaluated quantitatively using conventional toxicity assays, the 3D model showed lower toxicity and correlated well with animal studies reported in the literature.

Such 3D cell culture models have several advantages in that they can be used for longterm testing of engineered nanoparticles, and for understanding processes at the tissue level. It is suggested that the $3 \mathrm{D}$ model can act to bridge $2 \mathrm{D}$ in vitro models with in vivo animal studies.

\section{BIOSENSORS}

\section{Catching your breath \\ Nano Lett. 4, 1362-1368 (2009)}

Trace organic compounds in our breath contain vital information about the inner workings of our bodies, but they can be difficult to detect. Now, Hossam Haick and colleagues at the Technion-Israel Institute of Technology have described how to identify signs of lung cancer and kidney disease in breath samples, using arrays of carbon nanotubes.

The Technion team built field-effect transistors from a random network of carbon nanotubes, functionalized them with organic films, and then exposed the transistors to simulated breaths that contained known biological markers for lung cancer and kidney disease. The transistors show large changes in conductance on exposure to polar molecules (molecules with an electric dipole) and, more importantly, a decrease in conductance in response to non-polar molecules. It is thought that the non-polar molecules scatter charge carriers when they are adsorbed by the transistors, and that they might also push the nanotubes apart.

This is the first time that a carbon nanotube transistor has been able to detect non-polar molecules. Moreover, the detector can be made more selective and sensitive to different molecules by coating the nanotubes in various organic films. In future such devices could offer cheap, noninvasive and almost instantaneous methods for diagnosing diseases.

The definitive versions of these Research Highlights first appeared on the Nature Nanotechnology website, along with other articles that will not appear in print. If citing these articles, please refer to the web version.

\section{Top down Bottom up}

\section{Magnetic attraction}

A new kind of magnetic tweezers can make torque measurements on single molecules.

Sean Sun, a professor of engineering at Johns Hopkins University, had what seemed to be a straightforward goal: to build a standard magnetic tweezers setup to measure the torsional rigidity of chromatin - a linear biomolecule that is similar to DNA. However, as it became clear that this would not be so straightforward, the project began to gain collaborators. Greg Bowman, an expert in chromatin biochemistry who had just arrived at Hopkins, was introduced to the project by Sun's graduate student, Alfredo Celedon. And when Sun realized that standard probes would not be sufficient, he contacted two other Hopkins professors: Peter Searson, who had worked on magnetic probes, and Denis Wirtz, who had experience building magnetic tweezers.

In the experiments the molecule of interest is strung between a substrate and a probe. The probe is rotated by a magnetic field, twisting the molecule. The size of the thermally induced fluctuations in the twist angle is used to calculate torque. The problem with the standard tweezers approach is that the probe is held too tightly. This constrains the minimum resolvable torque to about $40 \mathrm{pN} \mathrm{nm} \mathrm{-}$ too high to be useful for single DNA or chromatin molecules.

Sun and his collaborators were able to demonstrate a new kind of torsion measurement in which the probe is more weakly held by the magnetic field. Its resolution is better than $1 \mathrm{pN} \mathrm{nm}$, which allowed torque measurements to be made on a single chromatin fibre for the first time (Nano Lett. 9, 1720-1725; 2009).

One of the challenges in bringing the collaboration together, according to Sun, was getting people to step out of their comfort zone and interested in the problem. "Collaborations are successful if the people involved are not $100 \%$ occupied with advancing their own agenda and careers, but are genuinely interested in good science" he says. "There must also be a person that takes ownership, and provides the driving force to bring everything together." 